\section{International Scientific Journal Theoretical \& Applied Science}

\author{
p-ISSN: 2308-4944 (print) e-ISSN: 2409-0085 (online) \\ Year: $2016 \quad$ Issue: 12 Volume: 44 \\ Published: $30.12 .2016 \quad \underline{\text { http://T-Science.org }}$
}

Sergey Alexandrovich Mishchik Associate Professor, Candidate of Pedagogical Science, Corresponding member of International Academy TAS, Assistant professor Department of Physics, State Maritime University Admiral Ushakov, Russia sergei_mishik@mail.ru

SECTION 21. Pedagogy. Psychology. Innovation in Education.

\title{
PRESENTATION OF THE TRAINING INFORMATION IN CONTROL ERTSGAMMING PEDAGOGOMETRIC RESEARCH VEHICLE EDUCATIONAL FACILITIES
}

\begin{abstract}
The main directions of presentation of educational information at ertsgamming control pedagogometric study transport of educational facilities in the formation of the mathematic model of educational activity on the nature of achievement criteria vital activity, cycling, systematic and phasing, which form the basic cell of the transport education space, as well as the use of the twelve pointed star Ertsgammy relative-enforcement of the principle of representation ertsgamming, which will determine the bases pedagogometric through formation substantive methods hyperspace professional transport vital activity, psychological and educational activity theory, psycho-pedagogical system analysis and the theory of the formation of mental actions.

Key words: educational information, educational facility, pedagogometric, analysis, transport livelihoods, cyclical, systemic, stages, the principle ertsgamming, star Ertsgammy.

Language: Russian

Citation: Mishchik SA (2016) PRESENTATION OF THE TRAINING INFORMATION IN CONTROL ERTSGAMMING PEDAGOGOMETRIC RESEARCH VEHICLE EDUCATIONAL FACILITIES. ISJ Theoretical \& Applied Science, 12 (44): 44-49.

Soi: http://s-o-i.org/1.1/TAS-12-44-10 Doi: crossef http://dx.doi.org/10.15863/TAS.2016.12.44.10
\end{abstract}

УДК 372.851

\section{ПРЕДСТАВЛЕНИЕ УЧЕБНОЙ ИНФОРМАЦИИ ПРИ ЭРЦГАММНОМ КОНТРОЛЕ ПЕДАГОГОМЕТРИЧЕСКОГО ИССЛЕДОВАНИЯ ТРАНСПОРТНЫХ ОБРАЗОВАТЕЛЬНЫХ ОБЪЕКТОВ}

Аннотация: Рассмотрены основные направления представления учебной информации при эригаммном контроле педагогометрического исследования транспортных образовательных объектов при формировании математических моделей учебной деятельности относительно характера достижения критериев жизнедеятельности, ичиклиности, системности и этапности, которые образуют базисную ячейку транспортного образовательного пространства, а также применение двенадцати конечной звезды Эригаммы относительно представления принципа эризгаммности, который определит основы педагогометрики через формообразование предметными методами гиперпространства профессиональной транспортной жизнедеятельности, психолого-педагогической теории деятельности, психологопедагогического системного анализа и теории формирования умственных действий.

Ключевые слова: учебная информация, образовательный объект, педагогометрика, анализ, транспортная жизнедеятельность, иикличность, системность, этапность, принции эризгаммности, звезда Эризгаммы.

\section{Introduction}

Представление учебной информации при эрцгаммном контроле педагогометрического исследования транспортных образовательных объектов при формировании математических моделей учебной деятельности относительно характера достижения критериев жизнедеятельности, цикличности, системности и этапности, которые образуют базисную ячейку транспортного образовательного пространства. Это проявляется в организации контрольной деятельности относительно уровня

ISPC Generalization of scientific results, 
представления в учебной информации: базисной звезды Эрцгаммы гиперпространства жизнедеятельности (Е1); базисного целостносистемного цикла жизнедеятельности (Е2); базисной звезды Эрцгаммы системного анализа (Е3); базисного проявления двенадцати этапов и форм познавательного гиперпространства жизнедеятельности относительно образовательного процесса (Е4) [1].

Каждая учебная информация с признаком базисно-нормативной эрцгаммности, независимо от целевого назначения, выполняет собственную функцию психолого-математического представления, имеющего соответствующий показатель базисно-нормативного целостного развития относительно характеристик собственной значимости [2]. Каждый базиснонормативный глобальный объект (E1N, E2N, E3N, E4N) образовательного пространства выполняет синфазно три сравнительные функции: ориентировки, исполнения и контроля собственной фазы развития образовательного процесса относительно нормативной учебнопрофессиональной деятельности эрцгаммного типа. Поэтому можно организовать представление учебной информации при эрцгаммном контроле педагогометрического исследования транспортных образовательных объектов, выражающего степень многофазного отклонения всех составляющих процессов развития студентов [3].

\section{Materials and Methods}

Представление учебной информации при эрцгаммном контроле педагогометрического исследования транспортных образовательных объектов при формировании математических моделей учебной деятельности относительно характера достижения критериев эрцгаммности на различных целостно-системных представлениях о профессиональной практической деятельности связывается с различными информационными представлениями об ориентировочных, исполнительных и контрольных качествах транспортных процессов [4].

Системность динамической прикладной физики морского флота проявляется в применении действий системного анализа. Базисной характеристикой динамических параметров судна являются координаты, скорость, ускорение и действующие силы при его движении [5].

Способность судна двигаться в окружающей среде с заданной скоростью при определенной мощности главных двигателей является ходкость. Судно движется на границе двух сред — воды и воздуха. Плотность воды в 800 раз больше плотности воздуха поэтому сопротивление воды больше воздушного.

Вследствие вязкости воды между корпусом судна и ближайшими к корпусу слоями воды возникают силы сопротивления трения $\boldsymbol{R}_{\boldsymbol{T}}$. Сопротивление трения зависит от скорости, от смоченной поверхности корпуса судна и от степени шероховатости. Сопротивление трения определяют расчетным путем.

При обтекании корпуса судна вязкой жидкостью происходит перераспределение гидродинамических давлений по его длине. Равнодействующая этих давлений является сопротивлением формы $\boldsymbol{R}_{\boldsymbol{\alpha}}$. Сопротивление формы зависит от скорости судна и от его формы. Волновое сопротивление $\boldsymbol{R}_{B}$ возникает из-за образования волн в зонах повышенного и пониженного давления при движении судна. Сопротивление выступающих частей $\boldsymbol{R}_{\boldsymbol{B} \Psi}$ зависит от сопротивления трения и от формы выступающих частей. Воздушное

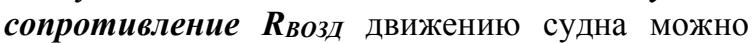
определить путем продувки надводной части модели в аэродинамической трубе.

Тогда полное сопротивление движению судна определяется как сумма отдельных составляющих

$$
\begin{aligned}
& \boldsymbol{R}=\boldsymbol{R}_{\Phi}+\boldsymbol{R}_{\boldsymbol{T}}+\boldsymbol{R}_{\boldsymbol{B}}+\boldsymbol{R}_{\boldsymbol{B}}+\boldsymbol{R}_{\text {возд }} \\
& \text { Это } \quad \text { сопротивление }
\end{aligned}
$$

является буксировочным, так как оно равно усилию в тросе, возникающему при буксировке судна. Мощность, необходимая для буксировки судна со скоростью $v$, есть буксировочная мощцость (кВт или л. с):

$E P S=R v / 102$ или $E P S=R v / 75$,

где $\boldsymbol{R}$ - полное сопротивление, $\boldsymbol{H} ; \boldsymbol{v}-$ скорость судна, $\boldsymbol{M} / \boldsymbol{c}$.

Мощность на фланце главного двигателя больше буксировочной, так как при ее определении необходимо учитывать пропульсивный коэффициент $\boldsymbol{\eta}\left(\boldsymbol{\eta}=\boldsymbol{\eta}_{\boldsymbol{P}} \boldsymbol{\eta}_{\boldsymbol{K}}\right.$, где $\boldsymbol{\eta}_{\boldsymbol{P}}-$ коэффициент полезного действия движителя, а $\boldsymbol{\eta}_{\boldsymbol{K}}$ - коэффициент влияния корпуса), КПД редуктора $\boldsymbol{\eta}_{\boldsymbol{P}}$ д, КПД валопровода $\boldsymbol{\eta}_{\boldsymbol{B}}: \boldsymbol{N}_{\boldsymbol{e}}=\boldsymbol{E P S} / \boldsymbol{\eta}$ ПРЕД $\boldsymbol{\eta}_{\boldsymbol{B}}$

Скорость судна на волнении уменьшается. Поэтому на судах мощность двигателя увеличивают с целью получения заданной скорости на определенном волнении [6].

В процессе движения судна возникает проблема управляемости, которая определяет способность судна быть поворотливым и устойчивым на курсе. Поворотливость - есть способность судна подчиняться действию руля, а устойчивость на курсе - способность сохранять заданное направление движения. Способность судна самопроизвольно отклоняться от курса под влиянием внешних сил является рыскливость. 
Для обеспечения требуемой управляемости в кормовой части судна устанавливают один или несколько рулей. Если на движущемся со скоростью $\boldsymbol{v}$ судне переложить руль на угол $\boldsymbol{\alpha}$, то на одну сторону руля начнет действовать давление набегающего потока воды равнодействующая гидродинамических сил $\boldsymbol{P}$, приложенная в центре давления и направленная перпендикулярно к поверхности руля. Приложим в центре тяжести судна взаимно уравновешенные силы $\boldsymbol{P}_{1}$ и $\boldsymbol{P}_{2}$, равные и параллельные $\boldsymbol{P}$. Силы $\boldsymbol{P}$ и $\boldsymbol{P}_{2}$ образуют пару сил, момент которой $\boldsymbol{M}_{\boldsymbol{B} \boldsymbol{P}}$ поворачивает судно вправо, $\boldsymbol{M}_{\boldsymbol{B} \boldsymbol{P}}=\boldsymbol{P} \boldsymbol{\ell}$, где плечо пары $\boldsymbol{\ell}=\boldsymbol{G} \boldsymbol{A} \cos \boldsymbol{\alpha}+\boldsymbol{a}$.

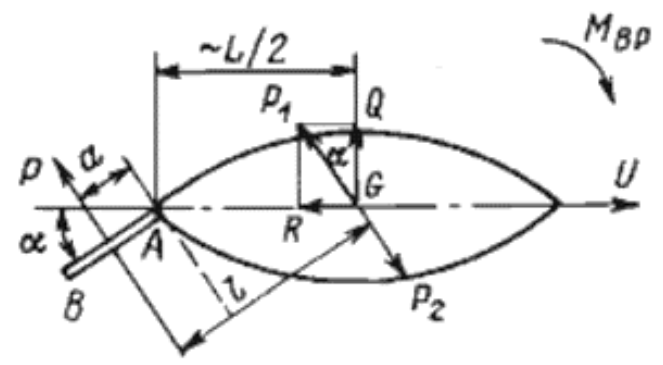

Рисунок 1 - Управляемость судна.

Центр тяжести судна находится на мидельшпангоуте, а величина $\boldsymbol{a}$ мала. Тогда $\boldsymbol{G A}=\boldsymbol{0 , 5 L}$; $\boldsymbol{\ell}=0,5 L \cos \alpha$ и $M_{B P}=0,5 P L \cos \alpha$. Силу $P_{1}$ разложим на составляющие $Q=P_{1} \cos \alpha=P \cos \alpha$ и $R=P_{1}$ $\sin \alpha=P \sin \alpha$. Сила $Q$ вызывает дрейф перемещение судна перпендикулярно к направлению движения, а сила $\boldsymbol{R}$ уменьшает его скорость.

Элементами циркуляции судна являются: $\boldsymbol{D}_{\boldsymbol{L}}$ - диаметр циркуляции; $\boldsymbol{D}_{\boldsymbol{T}}-$ тактический диаметр циркуляции; $\boldsymbol{\beta}$ - угол дрейфа. После перекладки руля на борт $\boldsymbol{Ц T}$ судна начнет описывать в горизонтальной плоскости кривую, постепенно переходящую в окружность циркуляцию. Диаметр окружности $\boldsymbol{D}_{\boldsymbol{L}},-$ есть диаметр циркуляции. Расстояние между ДП до начала циркуляции и после поворота судна на $180^{\circ}$ - тактический диаметр циркуляции $\boldsymbol{D}_{\boldsymbol{T}}$. Мерой поворотливости судна является отношение диаметра циркуляции к длине судна. Угол между ДП судна и касательной к траектории есть угол дрейфа $\boldsymbol{\beta}$ [7] .

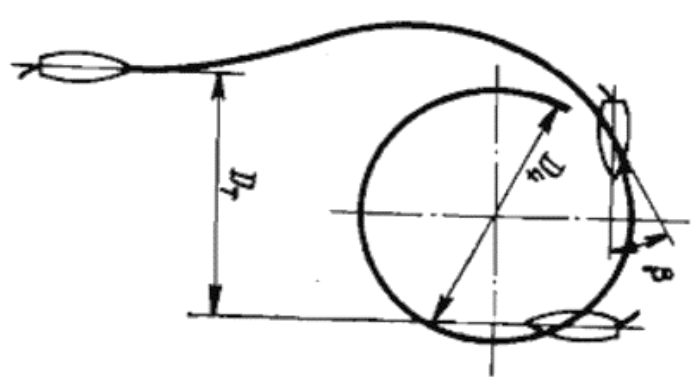

Рисунок 2 - Циркуляция судна.

При движении на циркуляции судно кренится на борт, противоположный перекладке руля, под действием центробежной силы инерции, приложенной в центре тяжести судна, и гидродинамических сил, приложенных к подводной части судна и рулю.

$$
X d x+Y d y+Z d z=\frac{1}{\rho}\left(\frac{\partial p}{\partial x} d x+\frac{\partial p}{\partial y} d y+\frac{\partial p}{\partial z} d z\right) .
$$

Системность гидравлической прикладной физики морского флота отражается в использовании действий системного анализа. Базисной характеристикой гидравлических параметров судна являются давление, объём и температура гидравлических рабочих элементов судовых механизмов [8].

Общим назначением гидравлической системы является передача энергии гидравлической жидкости по трубам с помощью насоса к гидравлическому мотору, который приводит в действие механизм.

Схема классической гидравлической системы определяется:

1 - гидравлический бак; 2 - клапан запорный; 3 - фильтр; 4 насос с электроприводом постоянной производительности и постоянным направлением потока жидкости; 5- предохранительный клапан; 6 - фильтр; 7 - гидрораспределитель с

ISPC Generalization of scientific results, 


\begin{tabular}{|c|c|c|c|c|c|c|}
\hline Impact Factor: & $\begin{array}{l}\text { ISRA (India) } \\
\text { ISI (Dubai, UAF } \\
\text { GIF (Australia) } \\
\text { JIF }\end{array}$ & $\begin{array}{l}=1.344 \\
=0.829 \\
=0.564 \\
=1.500\end{array}$ & $\begin{array}{l}\text { SIS (USA) } \\
\text { PИНЦ (Russia) } \\
\text { ESJI (KZ) } \\
\text { SJIF (Morocco) }\end{array}$ & $\begin{array}{l}=0.912 \\
=0.234 \\
=1.042 \\
=2.031\end{array}$ & $\begin{array}{l}\text { ICV (Poland) } \\
\text { PIF (India) } \\
\text { IBI (India) }\end{array}$ & $\begin{array}{l}=6.630 \\
=1.940 \\
=4.260\end{array}$ \\
\hline
\end{tabular}

местным ручным управлением рукояткой; 8 гидродвигатель; 9 - фильтр.

По конструктивному исполнению насос и гидродвигатель могут быть радиально- и аксиально-поршневые, шестеренные и винтовые.

пластинчатые,

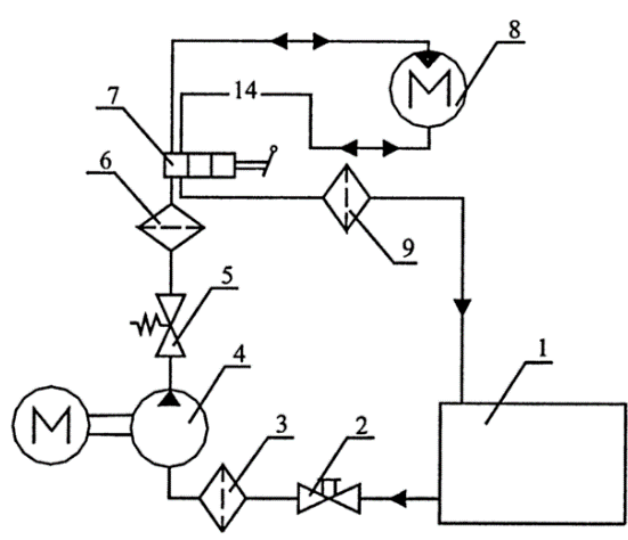

Рисунок 3 - Схема классической гидравлической системы.

На судах промыслового и морского флотов гидравлическую систему применяют для привода и управления рулевых машин, успокоителей качки, ВРШ, для грузовых, буксирных и ваерных лебёдок, брашпилей и шпилей, механизмов люковых закрытий, аппарелей и гидроподъёмников.

$$
\left.\begin{array}{r}
X-\frac{1}{\rho} \frac{\partial p}{\partial x}=0 \\
Y-\frac{1}{\rho} \frac{\partial p}{\partial y}=0 \\
Z-\frac{1}{\rho} \frac{\partial p}{\partial z}=0 .
\end{array}\right\}
$$

Дифференциальным равновесия жидкости являютс уравнения Л. Эйлера, которые описывают зависимость массовых и поверхностных сил от координат какой-либо точки покоящейся жидкости. В таком виде система уравнений была получена Л. Эйлером в 1775 году, которое можно представить в виде

Тогда можно определить основное уравнение гидростатики .

$$
p=p_{0}+\rho \cdot g \cdot h
$$

Давление в жидкости измеряется либо жидкостными приборами (пьезометром, вакуумметром, дифференциальным манометром), либо механическими манометрами.

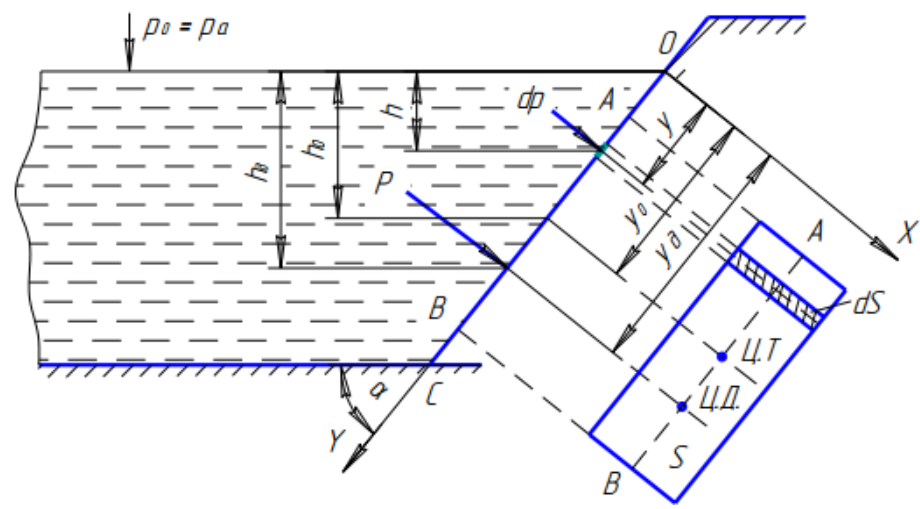

Рисунок 4 - Давление жидкости на наклонную поверхность

Давление жидкости на наклонную поверхность определяется силой гидростатического давления, действующей на смоченную часть поверхности $\mathrm{S}$ плоской стенки произвольной формы, характеризуется величиной, направлением и точкой приложения. Интеграл выражает статический момент площади фигуры АВ относительно оси X.

$$
\int_{S} y d S=y_{c} \cdot S
$$

Таким образом, величина абсолютного гидростатического давления равна произведению площади смоченной части плоской стенки на гидростатическое давление в центре тяжести.

ISPC Generalization of scientific results, 
Поэтому центр давления - определяет точку приложения равнодействующей избыточного гидростатического давления, что важно для определения размеров щитов, затворов и других сооружений. Для определения координат $\mathrm{dy}, \mathrm{dh}$ центра давления гидростатической силы воспользуемся теоремой Вариньона то есть, если произвольная система сил имеет равнодействующую, то момент этой равнодействующей относительно любой оси равен алгебраической сумме моментов всех сил этой системы :

$$
M_{P_{\text {uro }}}=\sum M_{i c}
$$

где МРизб - момент равнодействующей избыточной силы гидростатического давления относительно оси $\boldsymbol{X}$, а не абсолютного значения $\boldsymbol{P}$ потому, что координата приложения силы будет зависеть только от второй составляющей:

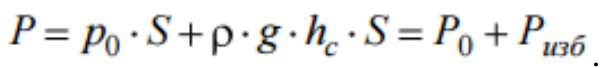

Давление жидкости на криволинейную поверхность в отличие от плоской стенки гидростатического давление в разных точках криволинейной стенки различается не только по величине, но и по направлению. Поэтому силу гидростатического давления, действующую на криволинейную стенку, непосредственно определить нельзя: ее находят через составляющие этого вектора. Рассмотрим криволинейную поверхность $\boldsymbol{A \boldsymbol { \sigma }}$, подверженную действию избыточного гидростатического давления - жидкость справа. Выделим площадку $\boldsymbol{d} \boldsymbol{S}$, центр тяжести которой погружен в жидкость на глубину $\boldsymbol{h}$. На площадку $\boldsymbol{d} \boldsymbol{S}$ будет действовать элементарная сила избыточного давления $\boldsymbol{d P}_{\boldsymbol{u з}}$ : $d P_{\text {изб }}=\rho \cdot g \cdot h \cdot d S$

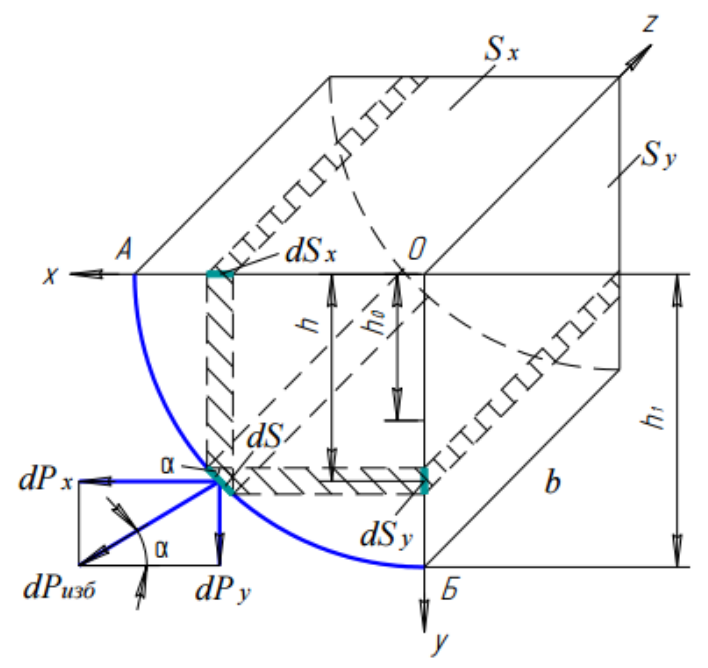

Рисунок 4 - Давление жидкости на криволинейную поверхность.

Объем $\boldsymbol{W}$, является суммой элементарных объемов выражается как тело давления. Тело давления - есть объем, ограниченный криволинейной поверхностью $\boldsymbol{A \boldsymbol { \sigma }}$, ее проекцией на уровень свободной поверхности и вертикальными плоскостями проецирования. Полная сила гидростатического давления определяется из выражения, $P_{\text {изб }}=\sqrt{P_{x}^{2}+P_{y}^{2}}$ где $\boldsymbol{P x}$ - горизонтальная составляющая силы избыточного гидростатического давления; $\boldsymbol{P \boldsymbol { y }}-$ вертикальная составляющая силы избыточного гидростатического давления. Направление полной силы Ризб определяется углом:

$$
\operatorname{tg} \alpha=\frac{P_{y}}{P_{x}}
$$

Полная сила избыточного гидростатического давления приложена в центре давления. Вектор полной силы давления Ризб должен проходить через точку пересечения ее горизонтальной и вертикальной составляющих, то есть $\boldsymbol{P} \boldsymbol{x}$ и $\boldsymbol{P} \boldsymbol{y}$, под углом[9]. Таким образом, центр давления для криволинейных поверхностей находится графоаналитическим путем. Если криволинейная поверхность цилиндрическая, то сила Ризб будет проходить через центр радиуса кривизны этой поверхности. При расчётах тонкостенных цилиндрических сосудов, подверженных внутреннему давлению: судотрубопроводы, котлы и ёмкости, заполненные жидкостью или газом - возникает задача расчета определения толщины их стенок.

Представленная учебная информация позволяет организовать полную форму эрцгаммного контроля образовательной деятельности относительно ориентировочной, исполнительной и контрольной составляющих всей системы комплексного созвездия квадроэрцгаммности [10]. 


\begin{tabular}{|c|c|c|c|c|c|c|}
\hline Impact Factor: & $\begin{array}{l}\text { ISRA (India) } \\
\text { ISI (Dubai, UAF } \\
\text { GIF (Australia) } \\
\text { JIF }\end{array}$ & $\begin{array}{l}=1.344 \\
=0.829 \\
=0.564 \\
=1.500\end{array}$ & $\begin{array}{l}\text { SIS (USA) } \\
\text { PИНЦ (Russia) } \\
\text { ESJI (KZ) } \\
\text { SJIF (Morocco) }\end{array}$ & $\begin{array}{l}=0.912 \\
=0.234 \\
=1.042 \\
=2.031\end{array}$ & $\begin{array}{l}\text { ICV (Poland) } \\
\text { PIF (India) } \\
\text { IBI (India) }\end{array}$ & $\begin{array}{l}=6.630 \\
=1.940 \\
=4.260\end{array}$ \\
\hline
\end{tabular}

\section{Conclusion}

Анализ основных направлений представления учебной информации при эрцгаммном контроле педагогометрического исследования транспортных образовательных объектов в процессе формирования математических моделей учебной деятельности относительно характера достижения критериев жизнедеятельности, цикличности, системности и этапности, которые образуют базисную ячейку транспортного образовательного пространства, определяет условия развития абсолютного образовательного цикла, отражающего специфическую структуру подготовки широкопрофильных транспортных специалистов при реализации международных образовательных стандартов.

\section{References:}

1. Mishchik SA (2014) Pedagogometrika and mathematical modeling educational activity. Materialy Mezhdunarodnoy nauchnoy konferenctsii "Modern mathematics in science" - 30.06.2014. ISJ Theoretical \&Applied Science 6(14): 54-56 Caracas, Venezuela. doi: http://dx.doi.org/10.15863/TAS.2014.06.14.10

2. Mishchik SA (2014) Simulation training activity methods of mathematical logic. Materialy Mezhdunarodnoy nauchnoy konferenctsii "Eurapean Science and Education" - 30.07.2014. ISJ Theoretical \&Applied Science 7(15): 72-74 Marseille, France. doi: http://dx.doi.org/10.15863/TAS.2014.07.15.13

3. Mishchik SA (2014) Mathematical modeling system integrity-cycle of life activity - first goal pedagogometriki. Materialy Mezhdunarodnoy nauchnoy konferenctsii "European Applied Sciences" - 30.08.2014. ISJ Theoretical \&Applied Science 8(16): 77-79. Aix-en-Provence, France. doi: http://dx.doi.org/10.15863/TAS.2014.08.16.13

4. Mishchik SA (2014) Mathematical modeling system integrity-curricular activities - the second problem pedagogometriki. Materialy Mezhdunarodnoy nauchnoy konferenctsii "European Innovation" - 30.09.2014. ISJ Theoretical \&Applied Science 9(17): 126-128 Martigues, France. doi: http://dx.doi.org/10.15863/TAS.2014.09.17.21

5. Mishchik SA (2014) Mathematical modeling holistic-systemic communicative activity - the third task pedagogometriki. Materialy Mezhdunarodnoy nauchnoy konferenctsii "European Scientific Achievements" 30.10.2014. ISJ Theoretical \&Applied Science 10(18): 45-47 Brighton, UK. doi: http://dx.doi.org/10.15863/TAS.2014.10.18.11
6. Mishchik SA (2014) Mathematical modeling integrity - system performance subject - fourth task pedagogometriki. Materialy Mezhdunarodnoy nauchnoy konferenctsii "Eurapean Science and Technology" 30.11.2014. ISJ Theoretical \&Applied Science 11(19): 51-54 Southampton, UK. doi: http://dx.doi.org/10.15863/TAS.2014.11.19.10

7. Mishchik SA (2015) Pedagogometrik - science and academic subject. Materialy Mezhdunarodnoy nauchnoy konferenctsii "European Technology in Science" 28.02.2015. ISJ Theoretical \& Applied Science 02 (22): 103-106 Malmö, Sweden. doi: http://dx.doi.org/10.15863/TAS.2015.02.22.17

8. Tokmazov GV (2014) Matematicheskoe modelirovanie $\mathrm{v}$ uchebno-professional'noy deyatel'nosti. Materialy Mezhdunarodnoy nauchnoy konferentsii «Modern mathematics in science» - 30.06.2014. ISJ Theoretical \& Applied Science 6(14): 44-46. - Caracas, Venezuela. doi: http://dx.doi.org/10.15863/TAS.2014.06.14.8

9. Tokmazov GV (2014) Analysis says study skills in the study of mathematics, Materialy Mezhdunarodnoy nauchnoy konferenctsii "Eurapean Science and Education" 30.07.2014. ISJ Theoretical \&Applied Science 7(15): 72-74 Marseille, France. doi: http://dx.doi.org/10.15863/TAS.2014.07.15.14

10. Tokmazov GV (2014) Mathematical modeling research skills in educational activity methods of probability theory. Materialy Mezhdunarodnoy nauchnoy konferenctsii "Eurapean Science and Technology" 30.11.2014. ISJ Theoretical \&Applied Science 11(19): 66-69 Southampton, United Kingdom. doi:

http://dx.doi.org/10.15863/TAS.2014.11.19.13 